\title{
IDEMPOTENTS IN BICATEGORIES
}

\author{
R. Paré, R. Rosebrugh and R.J: Wood
}

\begin{abstract}
It is shown that the category of fixed points of a left exact idempotent functor on a topos is again a topos. As well as a direct proof, a bicategorical proof is given which shows that the result only depends on certain bicategorical exactness properties.
\end{abstract}

\section{INTRODUCTION.}

The results presented here arose from the following observation and question by F.W. Lawvere. If $G$ is a graph, the category of sets with an action by $G$ (that is, diagrams $G \rightarrow$ set) is a very simply described example of a topos. In fact any presheaf topos is a geometric quotient of such a topos since any small category is a quotient of the free category on a graph. Since there is an inclusion from any Grothendieck topos to a presheaf topos, there is always a presentation of a Grothendieck topos as a "subquotient" of sets with action by a graph. Indeed, the topos so presented is exactly the objects fixed by the idempotent left exact functor on the sets with graph action obtained by using the direct and inverse images of the geometric morphisms in the presentation. The question which arises then is the following: if $\mathbf{E}$ is a topos and $F: \mathbf{E} \rightarrow \mathbf{E}$ is a left exact idempotent functor, is the category $\operatorname{fix}(F)$, whose objects and arrows are those fixed by $F$, again a topos?

In the next section we provide a positive answer. The question above involves left exact functors between toposes and these have been viewed as "progeometric morphisms" [5] and [6]. Moreover, our solution involves universal algebras for promonads (the left exact comonads) and idempotent procomonads (the idempotent left exact monads associated with the sheaf construction). The situation evoked is a bicategory equipped with a notion of proarrow and satisfying additional exactness axioms. In the third section we study the question of splitting idempotents (slightly generalised) in terms of universal algebras in a bicategory. In the last section we apply this to various proarrow equipments and show further that some of the required universal objects do not exist in the bicategory of categories and profunctors.

A few remarks on notation may be helpful. We use the standard () ${ }^{\text {OP }}$ and () ${ }^{\text {CO }}$ conventions. We write TOP for the bicategory of toposes and geometric morphisms;

Received 2 August, 1988

Research partially supported by grants from NSERC Canda.

Copyright Clearance Centre, Inc. Serial-fee code: $0004-9729 / 89 \quad \$ A 2.00+0.00$. 
TOPLEX for toposes, left exact functors and natural transformations. TOPLEX ${ }^{\mathrm{CO}}$ is the bicategory whose arrows are progeometric morphisms and which shares important exactness properties with PROF, the bicategory of categories and profunctors [10] and [11]. We assume the reader to be familiar with the basic facts about toposes and the theory of bicategories. Standard references are $[3,1]$ and $[8]$.

\section{IDEMPOTENT LEFT EXACT FUNCTORS}

In this section we prove the result about idempotent progeometric morphisms of toposes mentioned above. The notion of idempotent is first generalised slightly to a concept more appropriate to bicategories. An Idempotent (with capital I) is a functor $F: \mathbf{E} \rightarrow \mathbf{E}$ together with a natural isomorphism $\phi: F \stackrel{\cong}{\rightrightarrows} F^{2}$ such that $\phi F \cdot \phi=F \phi \cdot \phi$, that is, the following (coassociativity) diagram commutes:

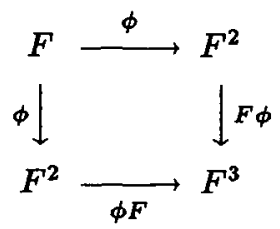

For example, if $H: \mathbf{E}_{0} \rightarrow \mathbf{E}$ and $G: \mathbf{E} \rightarrow \mathbf{E}_{0}$ are such that there is a natural isomorphism $\theta: 1_{\mathbf{E}_{0}} \cong G H$ then $(H G, H \theta G)$ is an Idempotent.

The category COALG $(F, \phi)$ has objects the pairs $(A, a)$ with $A$ in $\mathbf{E}$ and $a: A \rightarrow$ $F A$ subject to the requirement that

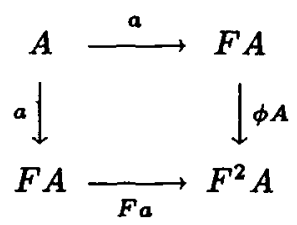

commutes. A morphism $f:(A, a) \rightarrow(B, b)$ in $\operatorname{COALG}(F, \phi)$ is a morphism $f: A \rightarrow B$ in $\mathbf{E}$ such that $b \cdot f=F f \cdot a$. The objects "fixed" by the Idempotent $(F, \phi)$ are those in the full subcategory $\operatorname{FIX}(F, \phi)$ of $\operatorname{COALG}(F, \theta)$ determined by the $(A, a)$ with a invertible. There is a functor $G: \mathbf{E} \rightarrow \operatorname{FIX}(F, \theta)$ defined by $G(A)=(F A, \phi A)$ and $G(f)=F f$. If $H: \operatorname{FIX}(F, \phi) \rightarrow \mathbf{E}$ is the forgetful functor, then $H G=F$ and $\theta: 1_{F I X(F, \phi)} \rightarrow G H$ defined by $\theta(A, a)=a$ is a natural isomorphism. Thus $G, H$ and $\theta$ provide a "Splitting" for $(F, \phi)$, a concept about which we will say more in the next section.

Before proceeding, we wish to remark tha if $F^{2}=F: \mathbf{E} \rightarrow \mathbf{E}$ is an idempotent, then $\operatorname{fix}(F)$ is equivalent to $\operatorname{FIX}\left(F, 1_{F}\right)$. Explictly, $A \mapsto\left(A, 1_{A}\right)$ and $(B, b) \mapsto F B$ provide an equivalence. 
Lemma 2.1. Let $\mathbf{E}$ be a topos and $(F, \phi)$ a left exact Idempotent on $\mathbf{E}$. The forgetful functor $U: \operatorname{COALG}(F, \phi) \rightarrow \mathbf{E}$ is cotripleable, and $\operatorname{COALG}(F, \phi)$ is a topos.

Proof: Standard arguments show that $\operatorname{COALG}(F, \phi)$ has all finite limits. Moreover, since $F$ is left exact, these are created by $U$ and so $U$ is cotripleable by the Crude Tripleability Theorem provided that it has a right adjoint. Define $R: \mathbf{E} \rightarrow$ $\operatorname{COALG}(F, \phi)$ on objects by $R A=\left(A \times F A, i_{A} \cdot\left\langle p_{2}, \phi A \cdot p_{2}\right\rangle\right)$ in which $p_{2}$ is the second projection from $A \times F A$ and $i_{A}: F A \times F^{2} A \cong F(A \times F A)$. $R A$ satisfies the coassociativity condition

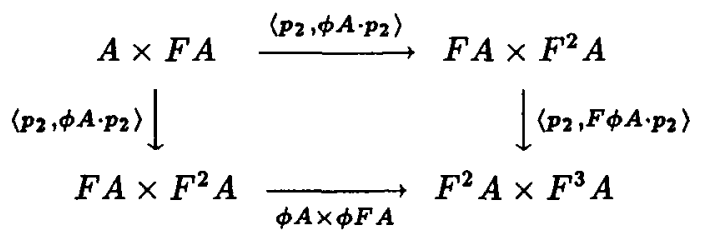

(in which we have suppressed mention of $i_{A}$ and $i_{F(A \times F A)}$ ) since $\phi$ is coassociative.

A morphism $(B, b) \rightarrow R A$ in $\operatorname{COALG}(F, \phi)$ is a commutive diagram

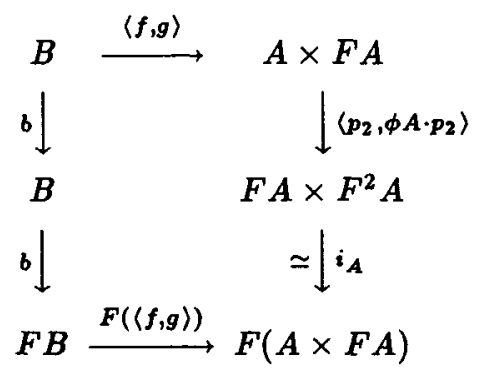

The commutativity of this diagram is equivalent to the condition $g=F f \cdot b$, so a morphism $\langle f, g\rangle:(B, b) \rightarrow R A$ is the same thing as a morphism $f: B \rightarrow A$, that is, $U \dashv R$. Thus $U$ is cotripleable and since it is also left exact, $\operatorname{COALG}(F, \phi)$ is a topos.

We note that the cotriple $U R=\mathbf{E} \times F$ is obtained by adjoining a counit to the already coassociative $(F, \phi)$.

LEMMA 2.2. Let $\mathbf{E}$ be a topos and $(F, \phi)$ a left exact Idempotent on $\mathbf{E}$. The full inclusion $J: \operatorname{FIX}(F, \phi) \rightarrow \operatorname{COALG}(F, \phi)$ has a left exact left adjoint.

Proof: Define $K: \operatorname{COALG}(F, \phi) \rightarrow \operatorname{FIX}(F, \phi)$ by $K(A, a)=(F A, \phi A)$ and $K f=F f$. Define a natural transformation $\eta: \operatorname{COALG}(F, \phi) \rightarrow J K$ by $\eta(A, a)=$ $a: A \rightarrow F A$ (well-defined since $F a \cdot a=\phi A \cdot a$ ) and another natural transformation $\varepsilon: K J \rightarrow \operatorname{FIX}(F, \phi)$ by $\varepsilon(A, a)=a^{-1}$ (well-defined since $F\left(a^{-1}\right) \phi A=a a^{-1}$ ). With these definitions we get commutativity of 


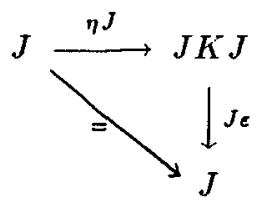

for it reduces to $a^{-1} a=A$ at the underlying object level. On the other hand $\varepsilon K \cdot K \eta$ reduces to $(\phi A)^{-1} \cdot F a$, which need not be an identity. However, $\varepsilon K \cdot K \eta$ is an idempotent transformation on $K$. Splitting $\varepsilon K \cdot K \eta$ as $L \leftrightarrows K$ provides a left adjoint to $J[4$, p.84], and since $K$ is left exact, so is $L$.

It is worth pointing out immediately that $\varepsilon K \cdot K \eta=K$ need not hold with the hypotheses of the lemma. Consider $F: \mathbf{E} \times \mathbf{E} \rightarrow \mathbf{E} \times \mathbf{E}, \mathbf{E}$ any topos, defined by $F(A, B)=(A, A)$ and $\phi: F \stackrel{\cong}{\rightrightarrows} F^{2}$ defined by the identity. An object in $\operatorname{COALG}(F, \phi)$ is an arrow $(f, g):(A, B) \rightarrow(A, A)$ such that $f^{2}=f$ and $f g=g$. Then

$$
\begin{aligned}
\epsilon K((A, B),(f, g)) \cdot K \eta((A, B),(f, g)) & =(A, A) \cdot(f, f) \\
& =(f, f) \neq(A, B) .
\end{aligned}
$$

Combining the Lemmas above we have:

Proposition 2.3. Let $\mathbf{E}$ be a topos and $(F, \phi)$ a left exact Idempotent on $\mathbf{E}$. Then $\operatorname{FIX}(F, \phi)$ is a topos.

Two points should be noted about the proof. First, consider the functor $S=$ $J K: \operatorname{COALG}(F, \phi) \rightarrow \operatorname{COALG}(F, \phi)$. This is a left exact functor with natural transformations $\eta$ : $\operatorname{COALG}(F, \phi) \rightarrow S$ and $\mu=J \varepsilon K: S^{2} \rightarrow S$ satisfying associativity for $\mu$ and $\mu \cdot \eta S=S$. However, $\mu \cdot S \eta=S$ is merely idempotent. The endofunctor which splits an idempotent natural transformation arising in this way is always a monad, and if $S$ is idempotent, so is the monad. Secondly, we have not only shown that $\operatorname{FIX}(F, \phi)$ is a topos, but we have done so by showing that it is a "subquotient" of $\mathbf{E}$, that is, there is an inclusion $\operatorname{FIX}(F, \phi) \rightarrow \operatorname{COALG}(F, \phi)$ to a topos which has a surjection from E. We will expand on the first point in the next section.

\section{IDEMPOTENT ARROWS IN A BICATEgoRY}

A monad on a category is a diagram in the bicategory, CAT, of categories and the category of algebras for the monad is a limit-like notion. The definitions make sense in any bicategory, $\mathcal{B}$, but if $\mathcal{B}$ is "concrete", for example $\mathcal{B}=\mathrm{CAT}$ or $\mathcal{B}=$ TOPLEX $\mathrm{CO}$, there may be no apparent advantage to considering universal properties. The previous section provides an efficient proof which settles a question for toposes and left exact functors. A mention of the univeral properties of $\operatorname{COALG}(F, \phi)$ and $\operatorname{FIX}(F, \phi)$ in the above may have been distracting, but.it is the point of this section that that proof is 
applicable very generally provided the universal properties are invoked. Applications are deferred until the last section.

For this section the reader is assumed to be somewhat familiar with universal properties in a bicategory. We give some standard definitions to set notation, and refer to $[4]$ and [7] for useful background material on monads.

If $A$ is an object in a bicategory $\mathcal{B}$, a monad on $A$ is a triple $\mathbf{t}=(t, \mu, \eta)$ where $t: A \rightarrow A$ is an arrow in $\mathcal{B}$, and $\mu: t^{2} \rightarrow t$ and $\eta: A \rightarrow t$ are transformations in $\mathcal{B}$ satisfying $\mu \cdot \mu t=\mu \cdot t \mu$ (associativity axiom) and $\mu \cdot \eta t=t=\mu \cdot t \eta$ (unitary axiom), that is, $(t, \mu, \eta)$ is a monoid in the monoidal category $\mathcal{B}(A, A)$ (with composition as tensor and identity on $A$ as unit). If $\mu$ is an isomorphism, call $\mathbf{t}$ an idempotent monad and recall that necessarily $\eta t=\mu^{-1}=t \eta$, so the definition of an idempotent monad need not involve $\mu$. A semimonad on $A$ is a pair $\mathbf{s}=(s, \mu)$ where $s: A \rightarrow A$, $\mu: s^{2} \rightarrow s$ and $\mu$ satisfies the equation (associativity) which makes $s$ a semigroup in the monoidal category $\mathcal{B}(A, A)$. If $\mu$ is an isomorphism, we call $\mathrm{s}$ an Idempotent on $A$. Every idempotent monad includes the data for an Idempotent, as does every idempotent comonad. Note that the example in the last section shows that an Idempotent need not arise in this way.

Let $\mathbf{e}=(e, \varepsilon)$ be an Idempotent on $A$ in $\mathcal{B}$. A splitting for $\mathbf{e}$ is the data $\operatorname{SPL}(\mathbf{e})$, $p, u$, isomorphisms $\sigma$ and $\tau$ as displayed:

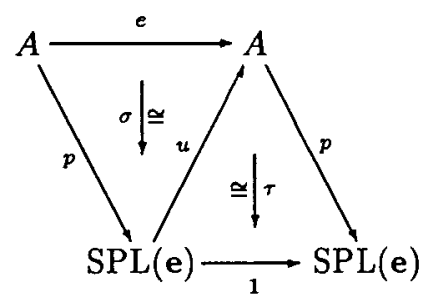

subject to the equation

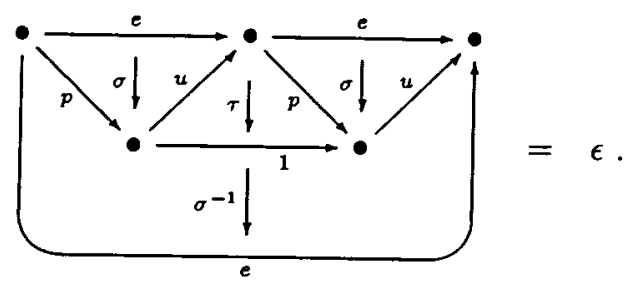

Clearly any homomorphism of bicategories $H: \mathcal{B} \rightarrow \mathcal{B}^{\prime}$ preserves splittings, so if Idempotents split in $\mathcal{B}^{\prime}$, then Idempotents split in $\mathcal{B}$ if and only if $H$ creates splittings. For an Idempotent $(F, \phi)$ in TOPLEX ${ }^{\mathrm{CO}}$ as in Section $2, \operatorname{FIX}(F, \phi)$ clearly provides 
a splitting in $\mathrm{CAT}^{\mathrm{CO}}$ after applying the forgetful homomorphism TOPLEX $\rightarrow$ CAT . Thus the question answered there, namely, "Is $\operatorname{FIX}(F, \phi)$ a topos?" is indeed generalised by "Does $\mathcal{B}$ admit splitting of Idempotents?".

For a monad $\mathrm{t}$ on $A$ a $\mathrm{t}$-algebra (with domain $X$ ) is a pair $(x, \xi)$ where $x: X \rightarrow$ $A$ and $\xi: t x \rightarrow x$ satisfying $\xi \cdot \eta x=x$ and $\xi \cdot \mu x=\xi \cdot t \xi$ (see $[4,7])$. If $(x, \xi)$ and $\left(x^{\prime}, \xi^{\prime}\right)$ are $t$-algebras with the same domain, a morphism $(x, \xi) \rightarrow\left(x^{\prime}, \xi^{\prime}\right)$ is a transformation $x \rightarrow x^{\prime}$ equivariant with respect to $\xi$ and $\xi^{\prime}$. Denote the resulting category $\operatorname{ALG}(X,(A, t))$ and, since the construction is evidently homomorphic in $X$, there is a homomorphism $\operatorname{ALG}(-,(A, t)): B^{O P} \rightarrow \mathrm{CAT}$. If this homomorphism is birepresentable, $A^{t}$ denotes the birepresenting object. It is called the Eilenberg-Moore object for $t$ or, as we shall call it here, the universal $t$-algebra.

$A t$-opalgebra for the monad $t$ is an algebra in $B^{O P}$, that is, a pair $(x, \xi)$ with $x: A \rightarrow X$ and $\xi: x t \rightarrow x$ satisfying "right-module" rather then "left-module" equations. The other duals of $\mathcal{B}$, namely $\mathcal{B}^{\mathrm{CO}}$ and $\mathcal{B}^{\text {COOP }}$ similarly enable the definition of coalgebra and opcoalgebra for a comonad on $A$ in $\mathcal{B}$.

For a semimonad $\mathbf{s}=(s, \mu)$ on $A$, it is natural to define an $\mathbf{s}$-algebra (with domain $X)$ as a pair $(x, \xi)$ with $x: X \rightarrow A$ and $\xi: s x \rightarrow x$ satisfying $\xi \cdot \mu x=\xi \cdot s \xi$. Deletion of requirements concerning the unit in the discussion about algebras for a monad gives us our definition of universal s-algebra, denoted by $A^{\mathbf{s}}$. Many similar variations are possible; we use one below.

For any of the above notions of algebra, we define a fix algebra of the same kind to be an algebra for which the structural transformation, denoted by $\xi$ above, is an isomorphism. Being a fix algebra is stable under composition, so we get a subhomomorphism, $\operatorname{FIX}(-,(A, t))$, of $\operatorname{ALG}(-,(A, t))$ whose value at $X$ is the category of fix algebras with domain $X$. A birepresenting object for $\operatorname{FIX}(-,(A, t))$ will be denoted by FIX(t). A universal fix opalgebra will be denoted by FIXOP(t). Fix algebras allow us to rephrase the splitting of Idempotents problem.

Proposition 3.1. For an Idempotent $\mathbf{e}=(e, \varepsilon)$ on $A$ in $\mathcal{B}$, if any one of FIX(e), $\operatorname{SPL}(\mathbf{e})$ or FIXOP(e) exists, then the others exist and all three are equivalent objects of $\mathcal{B}$.

Proof: Given $\operatorname{FIX}(\mathbf{e})=(u: \operatorname{FIX}(\mathbf{e}) \rightarrow A, \alpha: e u \stackrel{\cong}{\rightrightarrows} u)$, consider

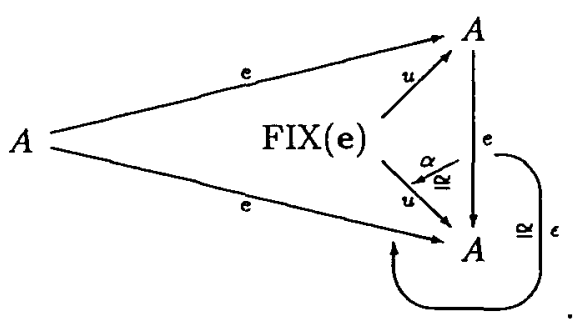




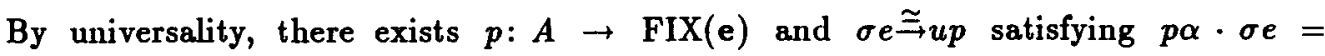
$\sigma \cdot \varepsilon: e^{2} \cong u p$. For such $p$ and $\sigma$, universality further ensures a unique $\tau: p u \rightarrow$ $1: \operatorname{FIX}(e) \rightarrow \operatorname{FIX}(e)$ satisfying $\alpha \cdot \sigma^{-1} u=u \tau: u p u \rightarrow u . \tau$ is necessarily an isomorphism and it is easy to verify that the splitting equation is satisfied for $\operatorname{FIX}(e), u, p$, $\sigma$ and $\tau$.

Given $\operatorname{SPL}(e), u, p, \sigma$ and $\tau$ as in the definition of splitting, consider,

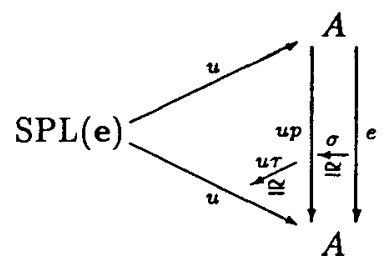

A routine calcuation shows that this defines a universal fix e-algebra.

The considerations for $\operatorname{FIXOP}(\mathbf{e})$ are dual.

For an idempotent monad $\mathrm{t}$, a pair $(x: X \rightarrow A, \xi: t x \rightarrow x)$ is a $\mathbf{t}$-algebra if and only if $\eta x$ is invertible and $\xi=(\eta x)^{-1}$. Thus, in this case $\operatorname{FIX}(t)$ and $A^{t}$ are equivalent objects in $\mathbf{B}$. For a general Idempotent, $\mathbf{e}=(e, \varepsilon)$, this is not the case, but suppose that $\left(v: A^{\bullet} \rightarrow A, \beta: e v \rightarrow v\right)$ is a universal e-algebra. Universality then gives a pair, $f, \phi$ as in

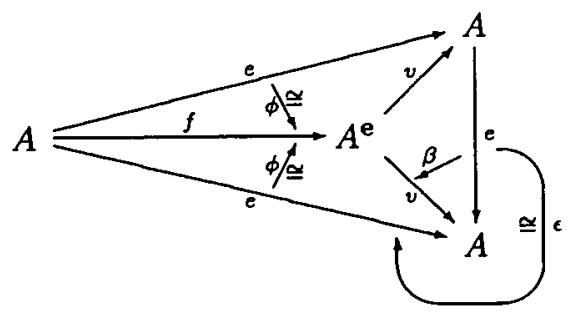

so that $(\beta f) \cdot(e \phi)=\phi \cdot \varepsilon$ (so $\phi$ is an isomorphism of e algebras from $(e, \varepsilon)$ to $(v f, \beta f)$ ).

Now consider the composite $f v: A^{\bullet} \rightarrow A^{\bullet}$. Transformations $f v \rightarrow f v f v$, respectively $f v \rightarrow 1$, are in bijective correspondence with homomorphisms $(v f v, \beta f v) \rightarrow$ $(v f v f v, \beta f v f v)$, respectively $(v f v, \beta f v) \rightarrow(v, \beta)$. We claim that $v f v \stackrel{\phi^{-1} v}{\longrightarrow} e v \stackrel{e^{-1} v}{\longrightarrow}$ $e^{2} v \stackrel{\phi^{2} v}{\longrightarrow} v f v f v$ and $v f v^{\phi^{-1}} \rightarrow e v \stackrel{\beta}{\rightarrow} v$ are homomorphisms. The first follows since $\varepsilon$ is an associative isomorphism ( $e$ is an Idempotent). The second follows since $\beta$ is an e-algebra. Denote $g=f v$ and $\delta: g \rightarrow g^{2}$, respectively $\gamma \cdot g \rightarrow 1$ for the transformations induced by the above homomorphisms. Since the first homomorphism is an 
isomorphism, $\delta$ is invertible. Moreover, associativity of $\varepsilon$ implies associativity of $\delta$ and $\gamma g \cdot \delta=g$. (To check the last equation, compose it with $v$, apply definitions and invoke faithfulness of composition with $v$.)

However, $g \gamma \cdot \delta$ need not equal $g$ (consider again the example from Section 2). Summarising, $\mathbf{g}=(g, \delta, \gamma)$ is an idempotent semicomonad with a right coidentity on $A^{\circ}$. We define a coalgebra for such a structure to be a pair $(z, \zeta)$ where $z: X \rightarrow A^{\circ}$ and $\zeta: z \rightarrow g z$ satisfy $\gamma z \cdot \zeta=z$ and $\delta z \cdot \zeta=g \zeta \cdot \zeta$. (This is the other variation of the $\mathbf{t}$-algebra notion we promised earlier.) A pair $(z, \zeta)$ is a fix $\mathbf{g}$-coalgebra if and only if $\gamma z$ is invertible and $\zeta=(\gamma z)^{-1}$. (Note that $\gamma z$ is invertible if and only if $\beta z$ is invertible, and the latter implies $e \beta z=\varepsilon v z$ which emables one to check the coassociativity.)

LEMMA 3.2. If $\mathcal{B}$ has universal algebras for semimonads and universal fix coalgebras for idempotent semicomonads with right coidentity, then Idempotents split in B.

Proof: Let $\mathrm{e}=(e, \varepsilon)$ be an Idempotent on $A$ and $(v, \beta)$, where $v: A^{\bullet} \rightarrow A$ and $\beta: e v \rightarrow v$, be universal as in the preceding discussion. For an arbitary $\mathbf{e}$-algebra $(x, \xi)$, define $y$ and $\eta$ by universality so that the following commutes:

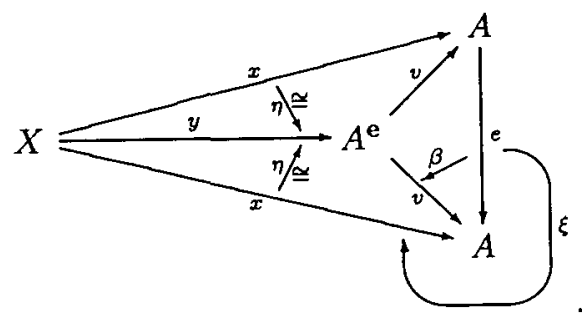

Now $(x, \xi)$ is a fix $\mathbf{e}$-algebra if and only if $\xi$ is an isomorphism if and only if $\beta y$ is an isomorphism if and only if $v \gamma y$ is an isomorphism if and only if $\gamma y$ is an isomorphism if and only if $\left(y,(\gamma y)^{-1}\right)$ is a fix $\mathbf{g}$-coalgebra. Hence we have an equivalence in $\mathcal{B}$, $\mathrm{FIX}(\mathbf{e}) \simeq \operatorname{FIXCO}(\mathbf{g})$.

The apparently unfamiliar hypotheses of Lemma 3.2 are fairly easy to satisfy in fanuliar terns.

LEммA 3.3. If $\mathcal{B}$ has (stable) local finite sums and universal algebras for monads, then $B$ has universal algebras for semimonads.

Proof: for $\mathrm{s}=(s, \mu)$ a semimonad on $A$, consider $t=A+s$ where " + " denotes 
the categorical sum in $\mathcal{B}(A, A)$. Define $\mu^{\prime}: t^{2} \rightarrow t$ to be the following composite:

$$
\begin{array}{r}
(A+s)(A+s) \cong A+s+s+s^{2} \longrightarrow A+s \\
{\left[\begin{array}{c}
i_{1} \\
i_{2} \\
i_{2} \\
i_{2} \mu
\end{array}\right]}
\end{array}
$$

Note that we use the full force of "local" here; the sums must be stable under composition from both sides. Define $\eta: A \rightarrow t$ to be the sum injection. Then $\mathbf{t}=\left(t, \mu^{\prime}, \eta\right)$ is a monad on $A$ and $A^{\mathrm{t}} \simeq A^{\mathrm{s}}$.

We remark that in checking the details of Lemma 3.3 one may find it convenient to treat first the case where $\mathcal{B}$ has but one object. For if $\mathcal{B}=(\mathcal{V}, \otimes, I, \ldots)$ is a monoidal category with finite sums over which $\otimes$ distributes, then $I++_{-}$provides a left adjoint to the forgetful functor from monoids in $\mathcal{V}$ to semigroups in $\mathcal{V}$. Moreover, for a semigroup $S$ in $\mathcal{V}$, actions $S \otimes X \rightarrow X$ are in bijective correspondence with unitary actions $(I+S) \otimes X \rightarrow X$. These facts are seen most easily using the language for monoidal categories developed by Jay [2].

Lemma 3.4. If $\mathcal{B}$ has splitting for local idempotents and universal algebras for idempotent comonads, then $\mathcal{B}$ has universal fix coalgebras for idempotent semicomonads with a right coidentity.

Proof: Let $\mathbf{g}=(g, \delta, \gamma)$ be an idempotent semicomonad with right coidentity on $A$. Then $g \gamma \cdot \delta$ is a local idempotent, that is, it is an idempotent in the category $\mathcal{B}(A, A)$. Let $g \rightarrow h \rightarrow g$ be a splitting for it, then $\delta$ and $\gamma$ induce an idempotent comonad structure on $h$. Call the comonad $h$, and then $\mathrm{FIXCO}(\mathrm{g}) \simeq A_{\mathbf{h}}$, the universal h-coalgebra.

The diagrams which establish these claims are tedious, but straight-forward. However, as with the previous lemma we can use Jay's language. It seems clearest to write the arguments for one-object $\mathcal{B}$ with "co" variance and use set-like notation.

First, let $(S, \cdot)$ be a semigroup with right identity 1 . Then left multiplication by 1 gives an idempctent on $S: 1(1 s)=(11) s=1 s$ for all $s \in S$. It is split by $M=\{s \in S \mid 1 s=s\}$, so $(M, \cdot, 1)$ is a monoid, even an ideal in $S$, that is, $M \otimes S \rightarrow M$ is a unitary action: $1(m s)=(1 m) s=m s$ for $m \in M, s \in S$. In fact, for every (unitary) action $X \otimes M \stackrel{\star}{\rightarrow} X$ there is a unique unitary action $X \otimes S \stackrel{\circ}{\rightarrow} X$ which restricts to $\star$. This follows from $x \circ s=(x \circ 1) \circ s=x \circ 1 s$ and $1 s \in M$.

Next, suppose that $S \otimes S \rightarrow S$ is an isomorphism. (The argument then making $S=\{1\}$ in set requires " $(s, t)=\left(s^{\prime}, t^{\prime}\right)$ implies $s=s^{\prime}$ and $t=t^{\prime}$ " which we do not have.) We have $s \otimes t=s t \otimes 1$ for all $s, t \in S$ and hence $M \otimes M \dot{\rightarrow} M$ and $M \otimes S \dot{\rightarrow} M$ 
are also isomorphisms. $M \otimes M \dot{\rightarrow} M$ being an isomorphisin implies that every (unitary) action $X \otimes M \stackrel{\star}{\rightarrow} X$ is an isomorphism. (For monads this is well-known.) In fact every unitary action $X \otimes S \stackrel{\circ}{\rightarrow} X$ is also an isomorphism. For any such action factors as

$$
\begin{gathered}
X \otimes S \rightarrow X \otimes M \otimes S \rightarrow X \otimes M \rightarrow X \\
x \otimes s \mapsto(x \otimes 1) \otimes s \\
x \otimes(m \otimes s) \mapsto x \otimes m s \\
x \otimes m \mapsto x \circ m
\end{gathered}
$$

in which each arrow is an isomorphism.

These arguments apply to $g$ and $h$ as above by showing that coalgebras

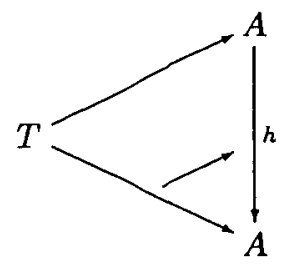

are the same thing as fix coalgebras

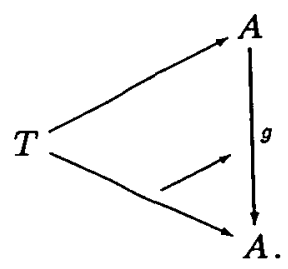

THEOREM 3.5. If $\mathcal{B}$ has local finite sums, splittings for local idempotents, universal algebras for monads and universal coalgebras for idempotent comonads, then Idempotents split in $\mathcal{B}$.

\section{Applications and counterexamples}

The applications we have in mind for Theorem 3.5 are to bicategories which are similar to TOPLEX ${ }^{\mathrm{CO}}$ in that

(i) hom categories are locally finitely cocomplete; 
(ii) finite collages exist, their injections are maps (that is, arrows with right adjoints), and the right adjoints of injections yield opcollages;

(iii) universal coalgebras exist for idempotent comonads.

Now for any proarrow equipment $\mathcal{K} \rightarrow \mathcal{M}$ satisfying the collage axiom (ii) (with collage injections in $\mathcal{K}$ ), Wood [11] showed that (ii) $\Rightarrow$ (i). Moreover, satisfying (i)-(iii) above implies Idempotents split by 3.5 .

Proposition 4.1. If $\mathcal{K} \rightarrow \mathcal{M}$ is a proarrow equipment satisfying the collage axiom and having universal coalgebras for idempotent comonads in $\mathcal{M}$, then Idempotents split in $\mathcal{M}$.

Corollary 4.2. Idempotents split in TOPLEX ${ }^{\mathrm{CO}}$, ABELLEX ${ }^{\mathrm{CO}}$ and LEX ${ }^{\mathrm{CO}}$.

Proof: The bicategories named (the latter two being Abelian categories and left exact functors and left exact categories and left exact functors) all are $\mathcal{M}$ 's for proarrow equipment as described.

Notably missing from the list above in 4.2 is PROF, the bicategory of categories and profunctors. It is well-known that PROF satisfies conditions i) and ii) above. Indeed its hom categories are small complete and cocomplete, and all right liftings and right extensions exist. By contrast iii) seems like a tame requirement. Universal algebras for idempotent comonads are absolute bilimits (preserved by all homomorphisms) and absolute bicolimits. Nevertheless, their existence distinguishes the examples listed above from PROF in favour of the former. We will exhibit counterexamples which establish this after first giving some further consideration to idempotent comonads. The right adjoint of a map $f: A \rightarrow B$ in a bicategory $\mathcal{B}$ is denoted by $f^{\star}$ and we write $\tilde{f}: A \rightarrow$ $f^{\star} f$ for the unit and $f: f f^{\star} \rightarrow B$ for the counit. If $\tilde{f}$ is an isomorphism, we call $f$ a proinclusion.

If $\mathbf{g}=(g, \delta, \varepsilon)$ is a comonad (not necessarily idempotent) on $B$ in $\mathcal{B}$, a universal coalgebra $(u, v)$ where $u: B_{\mathrm{g}} \rightarrow B$ and $v: u \rightarrow u g$, determines an equivalence of categories $\mathcal{B}\left(X, B_{\mathbf{g}}\right) \stackrel{\sim}{\rightarrow} \mathrm{ALG}(X,(B, \mathbf{g}))$ for all $X$ (by "composition with $(u, v)$ "). It follows immediately that $u$ is a map and $u^{\star}$ is a transpose of $(g, \delta)$ via the above equivalence. Necessarily there is an isomorphism of $\gamma: u u^{\star} \rightarrow g$ with $\varepsilon \cdot \gamma=u$. In fact, $\gamma$ must correspond, via adjointness, to $v$.

Returning to the case of $\mathbf{g}$ an idempotent comonad, we recall that $\delta$ is an isomorphism, equivalently $g \varepsilon=\varepsilon g$, and any $x: X \rightarrow B$ admits at most one coalgebra structure, namely $(\varepsilon x)^{-1}$ if $\varepsilon x$ is an isomorphism. In fact, $B_{\mathbf{g}}$ exists if and only if the inverter $\varepsilon: g \rightarrow B: B \rightarrow B$ exists, in which case they are equivalent objects of $\mathcal{B}$. Also $u^{\star}$ underlies a Kleisli object for $g$, and is a coinverter for $\varepsilon$. Most importantly for our present purposes, $u$ is a proinclusion. 
If $f: A \rightarrow B$ is any proinclusion, then $A$ is a universal coalgebra for the canonical idempotent comonad whose arrow is $f f^{\star}$. It follows that for $\mathbf{g}$ as above, if we have an isomorphism $\phi: f f^{\star} \cong$ $g$ with $\varepsilon \cdot \phi=f$ for some proinclusion $f: A \rightarrow B$, then $A$ is a universal coalgebra for $g$.

Thus, to show that a bicategory $\mathcal{B}$ has universal coalgebras for idempotent comonads is essentially to show that idempotent comonads are essentially coextensive with proinclusions.

For the remainder of this section we restrict our attention to $B=$ PROF. We should mention that we define $\operatorname{PROF}(\mathbf{A}, \mathbf{B})$ to be the category of functors $\mathbf{B}^{\mathrm{OP}} \times \mathbf{A} \rightarrow$ SET, and for a functor $F: \mathbf{A} \rightarrow \mathbf{B}$, the associated profunctor is denoted by $F_{\star}$, its right adjoint by $F^{\star}$. For a profunctor $f: \mathbf{A} \rightarrow \mathbf{B}$ we denote elements of $f(B, A)$ by wavy arrows $B \rightsquigarrow A$, and note that these have a left action by arrows of $\mathbf{B}$ and a right action by arrows of $\mathbf{A}$.

Now let $\mathbf{B}$ be a category and $\mathbf{g}=(g, \delta, \varepsilon)$ be an idempotent profunctor comonad. Assume further that $\mathbf{B}$ is Cauchy complete. This simplifies the discussion without any essential loss of generality since $\mathbf{B}$ and its Cauchy completion are equivalent in PROF. We write $\bar{\gamma}: B \rightarrow B^{\prime}$ for the straight arrow (in $\mathbf{B}$ ) associated with a wavy $\gamma: B \leadsto B^{\prime}$ by $\varepsilon$. The common value of $g \cdot \varepsilon$ and $\varepsilon \cdot g$ is a transformation $g^{2} \rightarrow g$ which provides

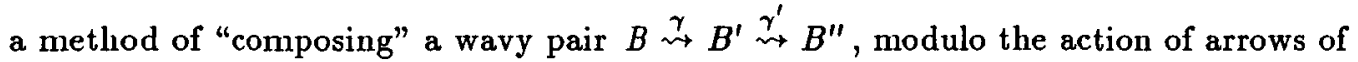
B. Write $\gamma \star \gamma^{\prime}$ for the result, and we obtain $\bar{\gamma} \gamma^{\prime}=\gamma \star \gamma^{\prime}=\gamma \overline{\gamma^{\prime}}$, associativity of $\star$, and $\left(\overline{\gamma \star \gamma^{\prime}}\right)=\bar{\gamma} \gamma^{\prime}$. Writing $\gamma_{0} \otimes \gamma_{1}$ for a pair which represents the effect of $\delta$ on $\gamma$, we get $\gamma_{0} \star \gamma_{1}=\gamma$ and if also $\gamma^{\prime} \star \gamma^{\prime \prime}=\gamma$, then $\gamma^{\prime} \otimes \gamma^{\prime \prime}=\gamma_{0} \otimes \gamma_{1}$.

It follows from the generalities above that if $\mathbf{B}_{\mathbf{g}} \rightarrow \mathbf{B}$ exists, it is PROF equivalent to the inclusion of a full subcategory.

LEMMA 4.3. For $\mathbf{B}, \mathbf{g}$ as above and $B \in \mathbf{B}$ the following are equivalent:

(i) $\varepsilon B_{\star}: g B_{\star} \rightarrow B_{\star}$ is an isomorphism;

(ii) $\forall X\left[\left(^{-}\right): g(X, B) \rightarrow \mathbf{B}(X, B)\right.$ is an isomorphism];

(iii) $\exists \iota: B \rightsquigarrow B$ such that $\bar{\imath}=B: B \rightarrow B$;

(iv) $\forall X\left[\left(^{-}\right): g(B, X) \rightarrow \mathbf{B}(B, X)\right.$ is an isomorphism];

(v) $B^{\star} \varepsilon: B^{\star} g \rightarrow B^{\star}$ is an isomorphism.

Define $\mathbf{B}(\mathbf{g})$ to be the full subcategory of $\mathbf{B}$ determined by those $B$ satisfying any (and hence all) of the above conditions. Note that this is a special case of $\mathbf{B}(\mathbf{g})$ as defined in Thiébaud $[\boldsymbol{\theta}]$.

LEMMA 4.4. If $\mathbf{B}_{\mathbf{g}}$ exists then it is given by $\mathbf{B}(\mathrm{g})$.

Proof: (Sketch) Since $\mathbf{B}_{\mathbf{B}} \stackrel{I_{*}}{\longrightarrow} \mathbf{B} \underset{\mathbf{B}}{\stackrel{g}{I_{g}}} \mathbf{B}$ is an inverter diagram in PROF, it follows 
that an object $\mathbf{1} \rightarrow \mathbf{B}_{\mathrm{g}}$ of $\mathbf{B}_{\mathbf{g}}$ gives rise to an object $B$ of $\mathbf{B}$ satisfying (i) above. On the other hand, such an object gives a profunctor $f: \mathbf{1} \rightarrow \mathbf{B}_{\mathbf{g}}$ with $I_{\star} f \cong B_{\star}$. Since $I_{\star}$ is a proinclusion, $f$ is a map. Since $\mathbf{B}$ is Cauchy complete and $\mathbf{B}_{\mathrm{B}}$, determined up to equivalence in PROF, may be taken to be a full subcategory closed under retracts, $f$ is isomorphic to an object of $\mathbf{B}_{\mathbf{g}}$.

For any idempotent profunctor comonad $\mathbf{g}$, write $J: \mathbf{B}(\mathbf{g}) \rightarrow \mathbf{B}$ for the inclusion. The $J_{\star} J^{\star}$ wavy arrows from $B$ to $B^{\prime}$ can be described as elements of the form $\beta_{0} \otimes \beta_{1}$ for some $B \stackrel{\beta_{0}}{\longrightarrow} A \stackrel{\beta_{1}}{\longrightarrow} B^{\prime}$ with $A$ in $\mathbf{B}(\mathrm{g})$. The $g$ wavy arrow $\iota: A \rightsquigarrow A$, for such $A$, as in (iii) of Lemma 4.3 , is unique. The assignment $\beta_{0} \otimes \beta_{1} \mapsto \beta_{0} \iota \beta_{1}$ determines a morphism of comonads $\kappa: J_{\star} J^{\star} \rightarrow \mathrm{g}$. Lemma 4.4 and the discussion above show that:

Proposition 4.5. $\mathbf{B}_{\mathbf{g}}$ exists if and only if $\kappa$ is an isomorphism.

Counterexample 4.6. Let $B$ be the monoid of non-negative rationals under addition. Let $g$ be the positive rationals with both left and right actions given by addition. Let $\varepsilon$ be the inclusion and for $\gamma$ in $g$ take $\delta(\gamma)$ to be the equivalence class of $(\gamma / 2, \gamma / 2)$ in $g^{2}$, which we denote by $\gamma / 2 \oplus \gamma / 2$ in this additive situation. Then $g=(g, \delta, \varepsilon)$ is an idempotent comonad on $B$ in PROF and $\mathbf{B}_{\mathbf{g}}$ does not exist.

Proof: Perhaps the only detail of the first assertion which requires comment is the equivariance of $\delta$. For $\beta$ in B and $\gamma$ in $g$,

$$
\begin{aligned}
\beta+(\gamma / 2 \oplus \gamma / 2) & =(\beta+\gamma / 2) \oplus \gamma / 2 \\
& =(\beta / 2+\gamma / 2+\beta / 2) \oplus \gamma / 2 \\
& =(\beta / 2+\gamma / 2) \oplus(\beta / 2+\gamma / 2) \\
& =(\beta+\gamma) / 2 \oplus(\beta+\gamma) / 2 .
\end{aligned}
$$

Now $\mathbf{B}(\mathbf{g})$ is the empty category since 0 , the identity in $\mathbf{B}$, is not in $g$. It follows that $J_{\star} J^{\star}$ is the empty module and not isomorphic to $g$.

Counterexample 4.7. Let $B$ be the partially ordered set of rationals with the usual order. Let $g$ be strict inequality on the rationals, $\varepsilon$ the inclusion and for $x<y$ in $g, \delta(x<y)=(x<(x+y) / 2) \otimes((x+y) / 2<y)$ in $g^{2}$. Then $g=(g, \delta, \varepsilon)$ is an idempotent profunctor comonad on $\mathbf{B}$ and $\mathbf{B}_{\mathbf{g}}$ does not exist.

Proof: As above, the first assertion follows if $\delta$ is well-defined. For $x^{\prime} \leqslant x<y$ we must show $\left(x^{\prime}<(x+y) / 2\right) \otimes((x+y) / 2<y)=\left(x^{\prime}<\left(x^{\prime}+y\right) / 2\right) \otimes\left(\left(x^{\prime}+y\right) / 2<y\right)$. This follows since $\left(x^{\prime}+y\right) / 2 \leqslant(x+y) / 2$. Again, $\mathbf{B}(\mathbf{g})$ is empty, making $J_{\star} J^{\star}$ the empty relation on the rationals and not isomorphic to $g$.

One could ask about the completeness of $\mathcal{V}-$ PROF for $\mathcal{V}$ other than a category of sets. Conceivably there exist suitable monoidal $\mathcal{V}$ for which $\mathcal{V}$-PROF admits all 
$\mathbf{B}_{\mathbf{g}}$. However, the second counterexample, in addition to being at the opposite extreme from the first, also shows $\mathcal{V}-\mathrm{PROF}$ to be incomplete for $\mathcal{V}=2$. We are indebted to G.M. Kelly for this observation.

\section{REFERENCES}

[1] J. Bénabou, 'Introduction to bicategories,', in Lecture Notes in Math 47, pp. 1-77 (Springer-Verlag, Berlin, Heidelberg, New York, 1967).

[2] C.B. Jay, 'Languages for monoidal categories', (preprint).

[3] P.T. Johnstone, Topos Theory (Academic Press, New York, 1977).

[4] S. Mac Lane, Categories for the Working Mathematician (Springer-Verlag, Berlin, Heidelberg, New York, 1971).

[5] R.D. Rosebrugh and R.J. Wood, 'Cofibrations in the bicategory of topoi', J. Pure Appl. Algebra 32 (1984), 71-94.

[6] R.D. Rosebrugh and R.J. Wood, 'Cofibrations II: left exact right actions and composition of gamuts', J. Pure Appl. Algebra 39 (1986), 283-300.

[7] R.H. Street, 'The formal theory of monads', J. Pure Appl. Algebra 2 (1972), 149-168.

[8] R.H. Street, 'Fibrations in bicategories', Cahiers de Topol. et Géom. Diff. 21 (1980), $111-160$.

[9] M. Thiébaud, Self-dual structure-semantics and algebraic categories, Thesis, Dalhousie University, 1971 .

[10] R.J. Wood, 'Abstract proarrows I', Cahiers de Topol. et Géom. Diff. 23 (1982), $279-290$.

[11] R.J. Wood, 'Proarrows II', Cahiers de Topol. et Géom. Diff. 26 (1985), 135-168.

Dr. R.J. Wood,

Dept of Math, Stats and Comp Sci,

Dalhousie University,

Halifax, Nova Scotia Canada. B3H 3J5

Dr R. Paré,

Dept of Math, Stats and Comp Sci,

Dalhousie University,

Halifax, Nova Scotia Canada. B3H 3J5
Dr R. Rosebrugh,

Department of Math and Computer Science,

Mount Allison University,

Sackville, New Brunswick E0A $3 \mathrm{CO}$

Canada. 